\title{
The Five Diaphragms in Patients Following Cardiac Surgery in Sternotomy: An Observational Study
}

\begin{abstract}
The recovery and rehabilitation process of patients following heart surgery in sternotomy is lengthy and requires significant time. This observational study on five diaphragms in patients undergoing a sternotomy is the first of its kind.

Objectives: Evaluate the position of the 5 bodily diaphragms, creating a platform of useful information for the practice of manual therapy. Understand if the diaphragm position coincides with the clinical status of the patient.
\end{abstract}

Setting: Department for Cardiac Rehabilitation, Foundation Don Carlo Gnocchi IRCCS.

Materials and methods: From January 2012 to July 2016, 300 cardiac patients underwent evaluation following a cardiothoracic intervention. In a supine position, palpation was conducted on the pelvic floor, the respiratory diaphragm, the thoracic outlet, the buccal floor and the cerebellar tentorium. The evaluation was undertaken on the first day of recovery in the ward. Rating scales were conducted such as the VAS, the walking test and the air life, an instrument for measuring the volume of air inhaled.

Results: Eleven different positional organisation structures of the 5 diaphragms have been highlighted which do not correspond to the patient's clinical status.

Conclusions: The data collected from the study allows us to obtain important information to guide the work of the manual therapist, but does not have a direct relationship with the patient's functional status.

Keywords: Osteopathic; Diaphragm; Manual therapy; Fascia; Phrenic nerve
Research Article

Volume 7 Issue 6 - 2017

\section{Bordoni $\mathrm{B}^{1 *}$, Marelli $\mathrm{F}^{2}$, Morabito $\mathrm{B}^{2}$ and} Sacconi B $B^{3,4}$

${ }^{1}$ Department of Cardiology, Institute of Hospitalization and Care with Scientific Address, Italy

${ }^{2}$ CRESO, School of Osteopathic Centre for Research and Studies, Italy

${ }^{3}$ Department of Radiological, Oncological and

Anatomopathological Sciences, Italy

${ }^{4}$ Center for Life Nano Science@Sapienza, Istituto Italiano di Tecnologia, Italy

*Corresponding author: Bordoni Bruno, Foundation Don Carlo Gnocchi IRCCS, Department of Cardiology, Institute of Hospitalization and Care with Scientific Address, S

Maria Nascente, Via Capecelatro 66, Milan 20100, Italy, Tel: 0039.02.3496300617; Email: bordonibruno@hotmail.com

Received: July 27, 2017 | Published: July 14, 2017

\section{Objectives}

It is estimated that each year over two million people worldwide undergo heart surgery in sternotomy, a surgical procedure which is not without iatrogenic complications $[1,2]$. These complications increase morbidity and mortality rates, slowing down the recovery process in patients [2]. In the 20 years of our clinic practice of cardiology, we have frequently encountered a plegia of the phrenic nerve, that may be transient, partially permanent or permanent, as found in published literature $[2,3]$. The lesion of the phrenic nerve may occur in $60 \%-85 \%$ of the population undergoing thoracic cardiac surgery, in particular the left nerve $[3,4]$. The reasons for this are not always clear. The most recognised is the lesion resulting from hypothermia during surgery to protect the myocardium, the collection of the internal mammary artery for the bypass (due to its close anatomical relationship with the phrenic nerve) and the ischemia caused by the sternal retractor as a result of stretching the nerve [4]. The respiratory diaphragm will be higher on the side of the lesion, and in diagnostic procedures, for example such as an x-ray, creating respiratory discomfort that can last for up to 1 year following surgery [4]. The vagus nerve may be subject to neurapraxia trauma from surgery, although the precise causes and the percentage in current literature are not present. The involvement of the vagus nerve causes oesophageal symptoms such as reflux or a pseudoangina chest pain [5-8]. With the opening of the chest, the vagus nerve and the recurrent of the vagus, in particular to the left (for its longer path) may be put under traction by the retractor, the same myocardial hypothermia induced by the surgeon may create a lesion [8]. The vagus nerve and the recurrent laryngeal nerve of the vagus may be damaged as a result of poor positioning of the patient on the operating table. Hyperextension or excessive lateral flexion of the upper cervical spine may adversely stretch the nerve, in rare cases even involving the hypoglossal nerve, resulting in motor issues of the tongue[8].

Other iatrogenic complications affecting the brachial plexus, identified in up to $37 \%$ of patients [9]. There are many hypotheses for why this may be the case. One of the most recognized causes is the use of the sternal retractor which, most likely, would cause a stretching of the ischemia to the plexus $[9,10]$.

The respiratory diaphragm has neurological and fascia connections such that any dysfunction of it will cause alterations not only locally but also systemically [11-13]. With current scientific knowledge, we can speak of the manual treatment of the 5 diaphragms: diaphragm muscle, pelvic floor, the buccal floor, the thoracic outlet and the tentorium of the cerebellum. It is possible to highlight the connections between all these structures, with fascial links and neurological continuity [14].

During correct respiration, or in the case of coughing or any other diaphragmatic physiological alteration, a symmetric change in the pelvic floor can be observed. For instance, if during inhalation the main inspiratory muscle descends, there will be 
a corresponding lowering of the pelvic floor [14]. The pelvic diaphragm not only plays a significant role in supporting the pelvic organs and in resisting increasing pressure, but it also affects the correct functionality of respiration [14]. The retroambigus nucleus - which is an important monitoring centre for phrenic medullary areas and is housed in the medulla oblongata or socalled bulb-controls the abdominal muscles as well [14]. 14 This means that respiration needs to be supported by the pelvic floor, in order to properly control the pressure of intra-abdominal liquid. It is probable that these same areas, which are connected to the motoneurons of the buccal floor, send the premotor order to the pelvic zone [14].

A dysfunction of the brachial plexus has a negative effect on both the function of the phrenic nerve and the diaphragm muscle, as the phrenic nerve is derived from the cervical root and brachial (C3-C5) $[14,15]$. The position of the same patient on the operating table, with the arm raised above his head, raises the first rib in elevation (left above), with the risk of compressing the phrenic nerve or breaking the rib.9 One cause of thoracic outlet syndrome is the elevation of the first rib and the tension of the scalene muscles, disturbing the smooth neuro-vascular passage of the brachial plexus [16].

The hypoglossal nerve receives a multitude of presynaptic impulses from the phrenic nerve and intercostal muscles. In regular respiration, the genioglossus and other muscles of the buccal floor, such as the hypoglossus, are electrically involved in coordination with the diaphragm, immediately before contraction of the diaphragm itself [15].

The phrenic nerve is anastomised with the vagus nerve at the cervical level; the latter anastomoses with the hypoglossal nerve [15]. The vagus nerve together with the hypoglossal nerve and Arnold's nerve (C2) innervate the dural area of the tentorium of the cerebellum, in the infratentorial portion [15].

The fascial continuum creates a structural continuity that gives form and function to every tissue and organ. The human body must be considered as a functional unit, where every area is in communication with another through the fascial continuum, consequently originating perfect tensegritive equilibrium $[17,18]$. The dural fascia system of the skull continues, coming into contact with the deep cervical fascia, continuing with the endothoracic fascia anteriorly and the thoracolumbar fascia posteriorly; the first continues with the transversalis fascia until the pubis, while the second involves the whole of the posterior area of the body [17]. This fascia system involves all the bodily diaphragms, including the respiratory diaphragm [15]. Trauma following a cardiac surgery with a sternotomy will affect all the diaphragms as a result of the anatomic and neurological continuity.

Understanding the position of the 5 diaphragms in cardiac surgery patients in sternotomy may be of assistance to manual therapists, helping to better organise the therapeutic process.

The palpation assessment has registered the tensional anomalies of the tissues of the mentioned body segments, indicating the preferential direction of the fascia or restrictions in movement [19]. In osteopathic and manual therapy practice, the discrimination of 5 diaphragms is performed by indicating the position of rotation or torsion of such bodily levels, based on simplified models of three-dimensional function according to the anatomical structures [19]. The authors of the study prefer to base manual evaluation on more realistic tensional anomalies rather than predetermined movement set out in osteopathic models. The authors believe that it is easier to conduct palpation on tensional tissue restrictions, giving an indication of the location of structures under examination [19]. Tissue alteration concerns the lack of pliability of the tissues on palpation, abnormal dragging of the fascia layers which can be assessed by positioning the hands and inducing a movement, or listening to how the structure is able to move; a painful area of the body, the hardness of the palpated segment on manual contact, the tensional vectors which, on palpation, do not have physiological anisotropy which is the basis of good fascia health $[19,20]$.

The objectives of the study were to evaluate the position of the bodily diaphragms, creating a platform of useful information to improve the practice of manual therapy. The task was also to understand if the diaphragm positioning coincides with the clinical status of the patient, as in osteopathy philosophy the position of the diaphragms has an effect on the state of the patient's health [19].

\section{Methods}

From January 2012 to July 2016, 300 cardiac patients underwent palpatory evaluation following a cardiothoracic intervention, on the first day of recovery to our cardiac rehabilitation department, Foundation Don Carlo Gnocchi IRCCS. The research is observational. All patients were informed and allowed the osteopathic evaluation voluntarily. The patient was never in danger during the evaluation; all patients of our clinic are evaluated and are subjected to standard treatment, according to the Declaration of Helsinki - Ethical Principles for Medical Research Involving Human Subjects. Written informed consent was obtained from all patients.

The criteria for inclusion in the study, in order to make the group as homogeneous as possible, required the presence of sternotomy cardiac surgery, with myocardial surgery such as a bypass, plastys or valve substitutions. The patients needed to be able to move autonomously from the bed and be collaborative with the study. All patients included in the study were 18 years old and above.

The exclusion criteria consisted in the lack of independence on the part of the patient, cardiac surgery in sternotomy for the removal of cardiac areas (myecotomy), the presence of a urinary catheter, previous intervention to the mediastinum. Patients with orthotopic heart transplant and with artificial heart support (L-VAD - Left-Ventricular Assistant Device) were excluded from the study. Patients who were under the age of 18 and those who had undergone a mini-thoracotomy were not included in the evaluation.

Some clinical data was considered for each manually assessed individual, such as the initial walking test (a routine test at our centre), pain assessment using a VAS - visual analogic scale, the functional capacity of the respiratory diaphragm using an inspiratory volume incentivator device (air life), able to indicate 
the litres of air inhaled; the subject's age, the level of haemoglobin and cardiac function (ejection frequency - EF) derived from the echocardiographic examination. Palpation was carried out while the patient was supine.

For the pelvic diaphragm the operator positioned his hands bilaterally on the iliac bones, resting on the anterior-superior iliac spine (Figure 1). Perpendicular pressure was applied towards the bed, to understand the elastic response of the tissues and whether or not there were preferential movement vectors. Once the active areas are assessed, the structure was listened to in order to understand how diaphragmatic breathing is reflected on the pelvic area, assessing the existence of possible caudal or cranial restrictions in the pelvic diaphragm area.

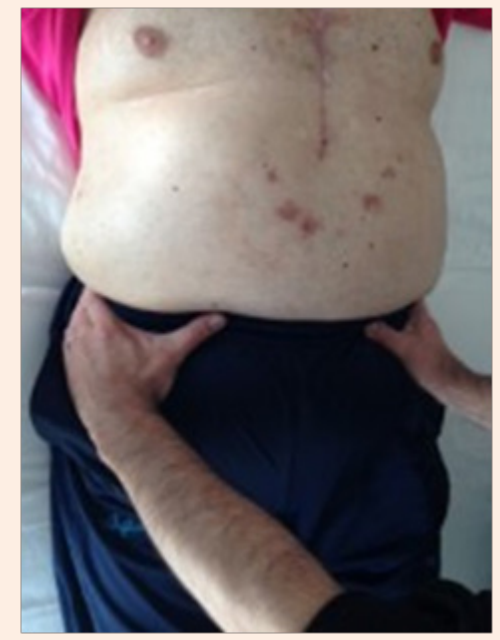

Figure 1: For the pelvic diaphragm the operator positioned his hands bilaterally on the iliac bones, resting on the anterior-superior iliac spine.

For the respiratory diaphragm, the operator has placed his hands on either side under the rib ramp with the thumbs under the costal arch and the remaining fingers on the ribs, orientating the fingers in line with the ribs (Figure 2). Small lateral offsets were actively induced to evaluate the elasticity of tissues and whether there were preferential movement vectors. Evaluating the respiratory diaphragmatic area using active stimuli, the structure was listened to if breathing had any cranial-caudal limitations, assessing the existence of possible restrictions.

For the, thoracic outlet area the operator placed his hands on both sides so that the index and middle involved the clavicle (the bone between two fingers), the thumb was directed toward the C7-D1 level, and the remaining fingers on the area under the clavicular area (Figure 3). The area was lightly evaluated with a slight caudal pressure and then, listening passively to understand how the body segment adapts to respiratory stress.

For the buccal diaphragm, the operator then positioned his fingers below and medially either side of the mandibular bone, starting from the gonion and including the entirety of the mandible (Figure 4). Applying light pressure to the cranial vector, the elasticity of the buccal floor was tested. In passive evaluation, always using the same palpatory approach, the operator assessed how the tissues behaved while the patient breathed.

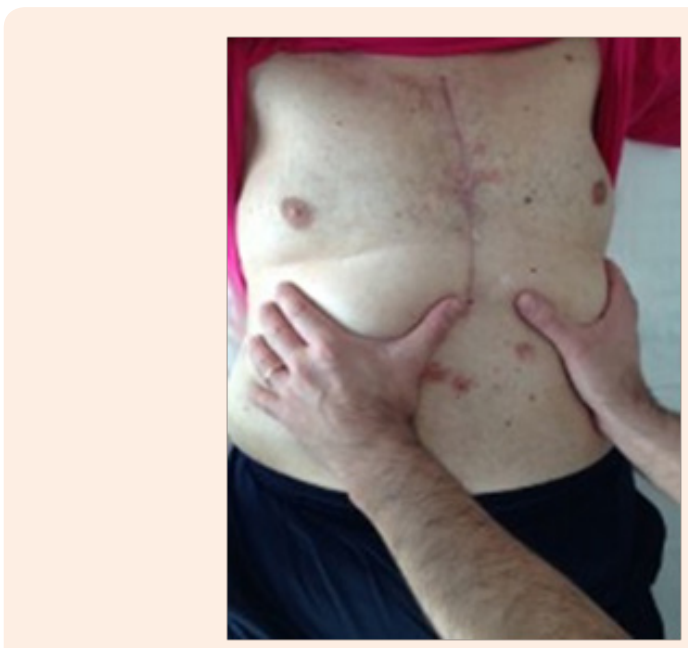

Figure 2: The operator has placed his hands on either side under the rib ramp with the thumbs under the costal arch and the remaining fingers on the ribs, orientating the fingers in line with the ribs.

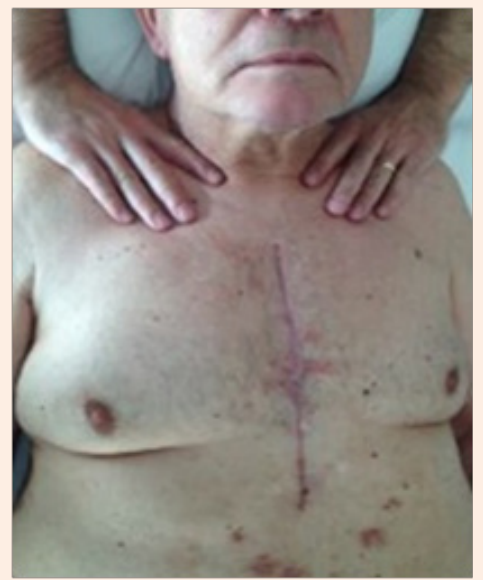

Figure 3: For the thoracic outlet area the operator placed his hands on both sides so that the index and middle involved the clavicle (the bone between two fingers), the thumb was directed toward the C7-D1 level, and the remaining fingers on the area under the clavicular area.

For the tentorial diaphragm or tentorium of the cerebellum, the operator's hands were positioned bilaterally with the little finger on the external occipital protuberance and the index the asterion (the meeting points between the occipital, temporal and parietal bones), following an imaginary line between these two reference points and providing supportalong the line between the two points with the ring finger and the middle finger, while the thumb did not participate in the listening exercise (Figure 5). The patient's head remains passive on the fingertips. The active assessment involved lightly pressing the fingers against the cranial points, looking for any painful areas or those which were particularly tight. Passive listening, using the same manual holding position, tried to assess 
the existence of possible restrictions or dragging of the fascia. The complete assessment for each patient took about 15 minutes. The days between the patients having undergone surgery and arriving in our department were a minimum of 4 days to a maximum of 12 .

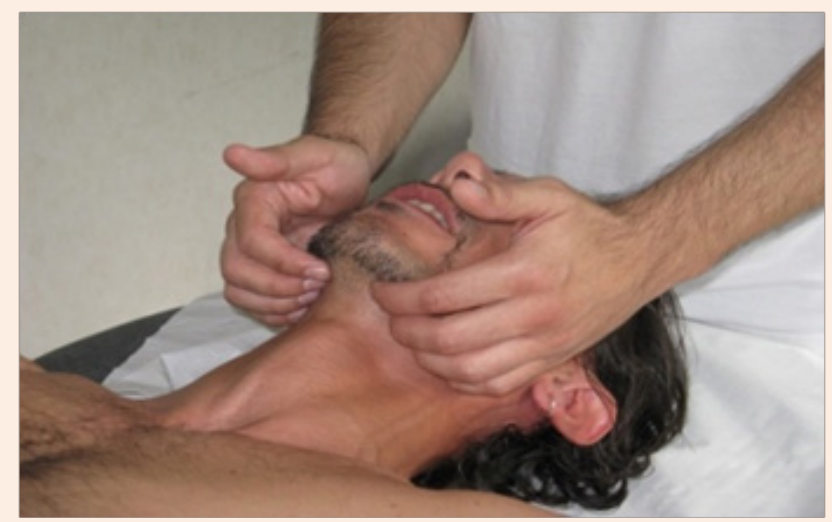

Figure 4: For the buccal diaphragm, the operator then positioned his fingers below and medially either side of the mandibular bone, starting from the gonion and including the entirety of the mandible.

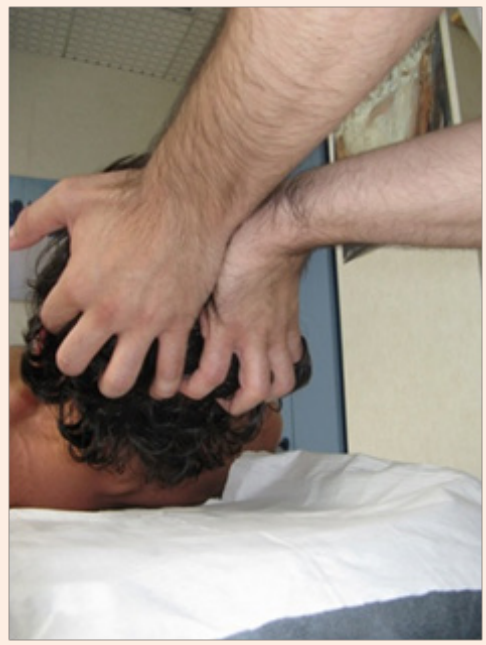

Figure 5: 101 patients presented with tensional vectors of the tentorium of the cerebellum and the buccal floor toward the right cranial area; the thoracic outlet and the respiratory diaphragm with tissue preference in the left cranial area; the pelvic floor displayed a preference for tensional vectors with the right cranial area.

\section{Results}

Palpatory assessment identified 11 positional structures. 101 patients presented with tensional vectors of the tentorium of the cerebellum and the buccal floor toward the right cranial area; the thoracic outlet and the respiratory diaphragm with tissue preference in the left cranial area; the pelvic floor displayed a preference for tensional vectors with the right cranial area. Figure 6 the patients undertook the walking test (WT) with an average distance covered of 350 meters; a VAS scale average of 5.9, average of 1250 litres of air assessed using the air-life, an average haemoglobin level of 9.1, an average age of 63, and an average value of the ejection frequency (EF) of $56 \%$.

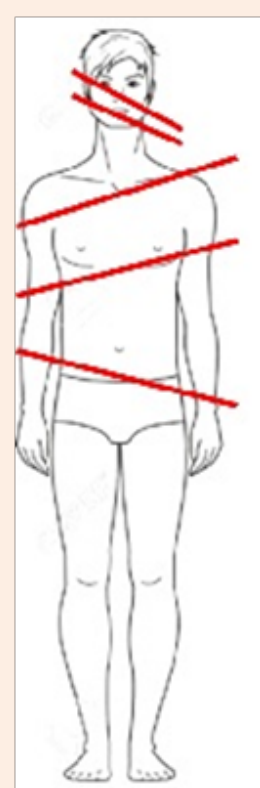

Figure 6: The second assessment concerned 47 patients having a tensional cranial preference of the tentorium of the cerebellum and the left side of the buccal floor; the thoracic outlet with restricted movement and cranial vectors to the right; the respiratory diaphragm with cranial vectors to the left; the pelvic floor with preferential vectors to the right cranial side.

The second assessment concerned 47 patients having a tensional cranial preference of the tentorium of the cerebellum and the left side of the buccal floor; the thoracic outlet with restricted movement and cranial vectors to the right; the respiratory diaphragm with cranial vectors to the left; the pelvic floor with preferential vectors to the right cranial side. Figure 7 the average WT was 330 metres; an average VAS of 5.7; an average of 1000 litres measured using the air life; average level of haemoglobin was 8.8; and average age of 65 years and an average EF of 54\%.

Another group of patients, a total of 33 individuals, presented with the tentorium of the cerebellum and the buccal floor displaying a preferential vector to the left; the thoracic outlet and respiratory diaphragm with a cranial and preferential tension to the right; the pelvic floor with a cranial tension to the left. Figure 8 the average distance covered with the WT was 335 metres, the VAS scale had an average of 6.3; the air life showed an average of 1250 litres; the average haemoglobin, age and EF were respectively 8,9 and $53 \%$; average age 69 .

Another positional structure was seeing in a group of 25 patients. The first four diaphragms had a preferential cranial vector to the left, while only the pelvic floor had a tendency towards a cranial preference to the right portion. Figure 9 the average WT was 327 metres; 6.4 for the VAS; 1250 litres measured using the air life; 9 on average for haemoglobin; an average age of 63 years and an average $\mathrm{EF}$ of $54 \%$ 


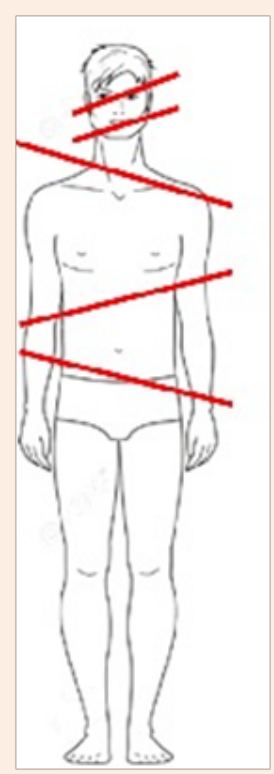

Figure 7: A total of 33 individuals, presented with the tentorium of the cerebellum and the buccal floor displaying a preferential vector to the left; the thoracic outlet and respiratory diaphragm with a cranial and preferential tension to the right; the pelvic floor with a cranial tension to the left.

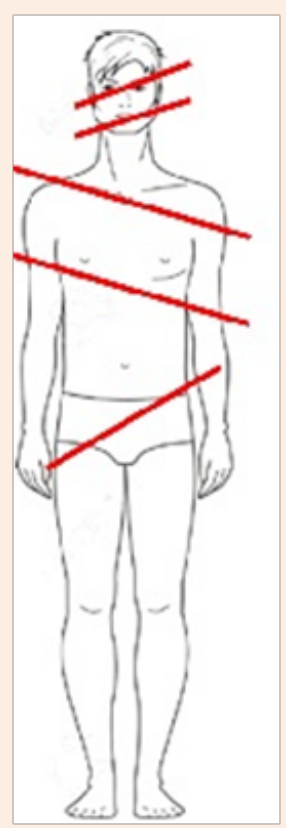

Figure 8: Another positional structure was see in a group of 25 patients. The first four diaphragms had a preferential cranial vector to the left, while only the pelvic floor had a tendency towards a cranial preference to the right portion.

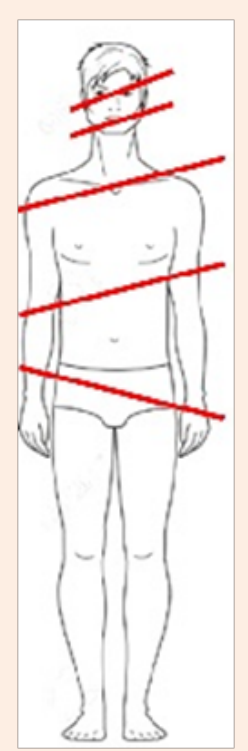

Figure 9: A group of 21 individuals had the tentorium of the cerebellum and the buccal floor with preferential cranial vectors towards the left section; the remaining diaphragms were bearing cranially towards the right.

A group of 21 individuals had the tentorium of the cerebellum and the buccal floor with preferential cranial vectors towards the left section; the remaining diaphragms were bearing cranially towards the right. Figure 10 the average WT was 360 metres; 6.2 for the VAS; 1000 litres on average measured using the air life; 9.1 on average for haemoglobin; an average age of 65 years and an average $\mathrm{EF}$ of $55 \%$

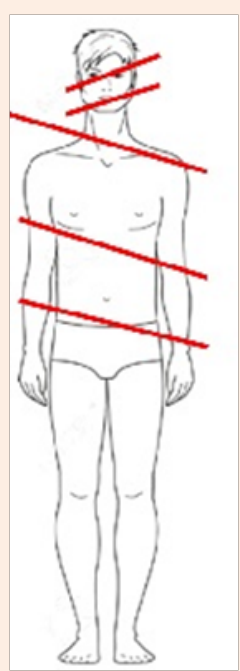

Figure 10: Another group of 19 patients presented with the first three diaphragms displaying a preferential cranial vector tension to the left; the respiratory diaphragm with a preferential cranial vector to the right; the pelvic diaphragm still tending cranially to the left. 
Another group of 19 patients presented with the first three diaphragms displaying a preferential cranial vector tension to the left; the respiratory diaphragm with a preferential cranial vector to the right; the pelvic diaphragm still tending cranially to the left. Figure 11 the average WT was 340 metres; 5.7 for the VAS; 1000 litres measured using the air life; 8.8 on average for haemoglobin; an average age of 49 years and an average EF of $54 \%$

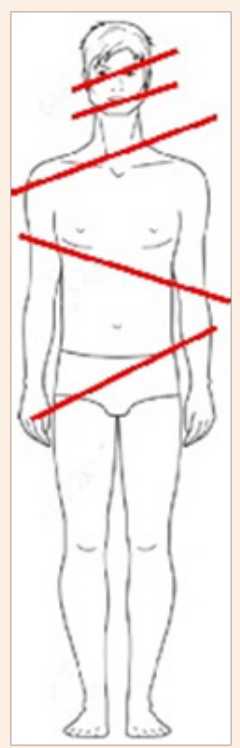

Figure 11: A group of 17 people presented with the first 4 diaphragms with a preferential cranial vector to the right and only the remaining pelvic floor with restricted movement and a preferential cranial vector to the left.

A group of 17 people presented with the first 4 diaphragms with a preferential cranial vector to the right and only the remaining pelvic floor with restricted movement and a preferential cranial vector to the left. Figure 12 the average WT was 345 metres; an average value of 6 for the VAS; 1000 litres measured using the air life; 8.9 on average for haemoglobin; an average age of 66 years and an average $\mathrm{EF}$ of $54 \%$.

A group of 14 patients were assessed with having a tentorium of the cerebellum with a preferential cranial vector to the right; the buccal floor with a preferential cranial direction to the left; the thoracic outlet had a preferential cranial direction to the right; the respiratory and pelvic diaphragms to the left and right respectively. Figure 8 the average WT was 348 metres; 5.7 for the VAS; 1250 litres measured using the air life; 9 on average for haemoglobin; an average age of 61 years and an average EF of $55 \%$.

Another group of 10 people registered diaphragm positions which were the exact opposite of the preceding group; the tentorium of the cerebellum with a preferential vector to the left: the buccal floor with a cranial preference to the right; the thoracic outlet, respiratory diaphragm and the pelvic floor to the left, right and left respectively. Figure 13 the average WT was 350 metres; 5.9 on average for the VAS; 1000 litres measured using the air life;
8.9 for haemoglobin; an average age of 58 years and an average EF of $53 \%$

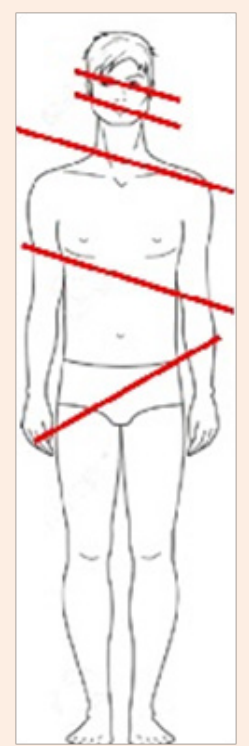

Figure 12: A group of 14 patients were assessed with having a tentorium of the cerebellum with a preferential cranial vector to the right; the buccal floor with a preferential cranial direction to the left; the thoracic outlet had a preferential cranial direction to the right; the respiratory and pelvic diaphragms to the left and right respectively.

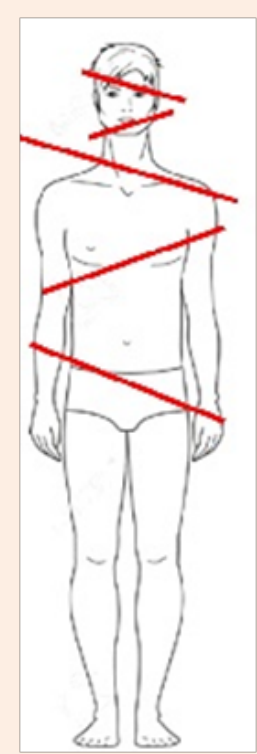

Figure 13: Another group of 10 people registered diaphragm positions which were the exact opposite of the preceding group; the tentorium of the cerebellum with a preferential vector to the left; the buccal floor with a cranial preference to the right; the thoracic outlet, respiratory diaphragm and the pelvic floor to the left, right and left respectively.

A group of 7 people had all the diaphragms cranially bearing to the right area. Figure 14 the average WT was 340 metres; 5.4 
on average for the VAS; 1250 litres measured using the air life; 9.1 on average for haemoglobin; an average age of 62 years and an average $\mathrm{EF}$ of $55 \%$.

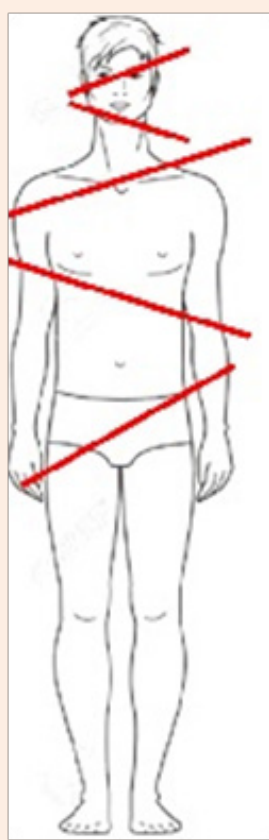

Figure 14: A group of 7 people had all the diaphragms cranially bearing to the right area.

The last group of patients, 6 individuals, registered through palpation that all the diaphragms had a preferential tension and cranial vector towards the left. Figure 15 the average WT was 355 metres covered; 5.9 on average for the VAS; 1000 litres measured using the air life; 9.2 on average for haemoglobin; an average age of 55 years and an average EF of $55 \%$.

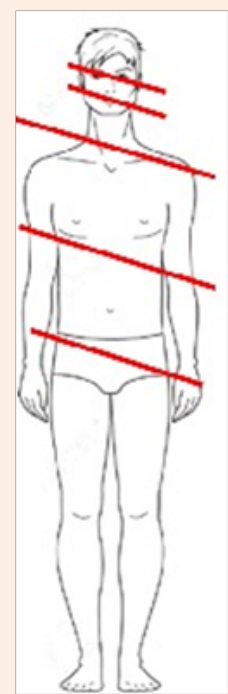

Figure 15: The last group of patients, 6 individuals, registered through palpation that all the diaphragms had a preferential tension and cranial vector towards the left.

\section{Discussion}

The osteopathic approach in the evaluation and treatment of the diaphragms of the body is very important. According to osteopathic medicine, positioning and the function that follows affects the general health of the patient, since according to the respiratory-circulatory model, a proper functional relationship of bodily diaphragms allows the immune and circulatory functions to influence the health of the patient [19]. Each area of the body that has restricted movement and a preferential fascial vector will have circulation and lymphatic stress disorder which will impact negatively on health [19]. According to the doctor and osteopath DO JG Zink, who studied in depth the treatment and assessment of diaphragms of the body, the patient's respiratory function has an effect on his health and on the clinical status; the treatment of the diaphragms will improve the clinical outcome and personal well-being, according to this approach.19 According to Zink, a person who presents with diaphragms of the body that are in perfect harmony and without dysfunction, these should all be in parallel, without preference or tissue restriction. Figure 16 in clinical practice by the same Zink, this perfection is excluded, as it is easier to find positional models that "compensate", in his view, that is to say diaphragms of the body with reciprocating positional vectors, or de-compeniate, where vectors are not reciprocating but have a disorganised structure [19,20].

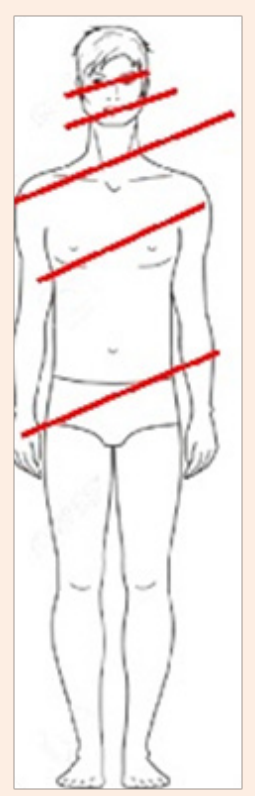

Figure 16: According to Zink, a person who presents with diaphragms of the body that are in perfect harmony and without dysfunction, these should all be in parallel, without preference or tissue restriction.

Current observational research took into consideration the 5 bodily diaphragms, while Zink's model is constructed on 4 diaphragms, without including the buccal floor. We believe our model is closer to modern scientific information $[14,15]$. We propose a hypothesis of manual evaluation protocol, as it is necessary to know precisely which is the anatomical area or areas that need more therapeutic attention. Probably, knowing in detail 
how to organize the conservative rehabilitation scheme, nonsurgical approach results could be further improved.

The results of our palpation and observation have highlighted 11 positional schemes of the diaphragms. The clinical and assessment data calculated on average for each scheme has not shown significant variability between the clinical and functional status of the patient and the arrangement of the bodily diaphragms. It suggests another consideration, namely, that the palpation of tissue which would determine the preferred cranial vector of the cerebellar tentorium has a high percentage of response with the position of the buccal floor. One third of patients, 101 people, have a preferential position as described in design.

Osteopathic Manipulative Treatment (OMT) could be a valid option as a complementary treatment of postoperative pain, intervening in the process that transforms nociceptive information into the subjective experience of pain. As a matter of fact, OMT has been proven effective in postoperative pain control after abdominal surgery [21]. A beneficial OMT effect was also observed on the recovery of patients after coronary artery bypass, improving also cardiac function as measured by the cardiac index $[22,23]$. Patients with musculoskeletal problems treated with OMT after elective knee or hip arthroplasty required less analgesia than the non treated patients [24]

We know that OMT has a positive effect on the system autonomy, improving cardiac response, such as blood frequency and pressure [25-27]. Osteopathic treatment improves the ability of blood vessels to maintain their thickness, making the contraction and passage of blood to the tissues more efficient [28]. This central and peripheral adaptation is able to put off cardio-vascular events [28]. OMT has been shown to improve the immune response, the lymphatic flow and to decrease the sympathetic tone in post-operative patients and in intensive therapy with possible therapeutic and preventative effects in patients [29].

We understand the strategic importance of the function of the diaphragm muscle on circulatory function and we know that if approached using manual techniques it can improve its function and excursions, with benefits to all bodily systems [30,31]. We know that the pelvic floor if treated using osteopathic methods, it's functional ability is improved [32]. The thoracic outlet with functional issues can be approached with osteopathic techniques, both in adults and as well as children [33,34]. Osteopathic techniques are able to influence the dural area, with palpatory quality that correctly identifies the variations derived from it, using precise rhythms [35-37].

It is important to remember that, as with other therapeutic techniques, whether manual or otherwise, conclusive scientific evidence is not available for every existing treatment; however, this does not mean that the therapy is invalid, as otherwise there would be no new developments or improvement in rehabilitative treatments starategies [15]. In this regard, we wish to reiterate that evidence-based medicine (EBM), which originated in the second half of the 19th century, is based on individual clinical expertise, best external evidence, patient values, and expectations: "External clinical evidence can inform, but can never replace, individual clinical expertise, and it is this expertise that decides whether the external evidence applies to the individual patient at all and, if so, how it should be integrated into a clinical decision[38]."

Palpation is an important tool to evaluate, classify and differentiate, with diagnostic capabilities; the fingertip has a tactile sensitivity that arrives to discern objects measured in micron [35-40]. The palpatory examination is able to report the position of the joints and possible altered joint movement, tissue abnormalities (temperature, tone, hardness, and etc.) from the surface to the depth of the various layers, and identify the painful anatomical area [40-42].

Having information available that patients undergoing cardiac surgery in sternotomy have fascia restrictions with preferential tensional vectors, will be of assistance to manual therapists when organising their therapeutic approach, in the knowledge that manipulation has a very positive impact on the health of the patient. Quite apart from the positional structure of the bodily diaphragms, which in this study has shown there is not a direct relation to the clinical and functional status of the patient, it is fundamental to release and improve the fascia restrictions in this specific population of patients.

\section{Conclusion}

The evaluation of osteopathic palpation in the 5 diaphragms of the body in 300 cardiac patients, who underwent cardio-thoracic surgery, has highlighted the structure of fascia restrictions and the preferential positioning of such structures. The observational study is the first of its kind in such a specific population of patients. Within the osteopathic approach, the diaphragms of the body are considered a pivotal point in the interpretation of the patient's clinical status and treatment. Our evaluation has not highlighted any relationship between the positional structure and the clinical and functional values of the patients.

\section{References}

1. van Gulik L, Janssen LI, Ahlers SJ, Bruins P, Driessen AH, et al. (2011) Risk factors for chronic thoracic pain after cardiac surgery via sternotomy. Eur J Cardiothorac Surg 40(6): 1309-1313.

2. Christensen JD, Seaman DM, Washington L (2014) Imaging of complications of thoracic and cardiovascular surgery. Radiol Clin North Am 52(5): 929-959.

3. Kodric M, Trevisan R, Torregiani C, Cifaldi R, Longo C, et al. (2013) Inspiratory muscle training for diaphragm dysfunction after cardiac surgery. J Thorac Cardiovasc Surg 145(3): 819-823.

4. Aguirre VJ, Sinha P, Zimmet A, Lee GA, Kwa L, et al. (2013) Phrenic nerve injury during cardiac surgery: mechanisms, management and prevention. Heart Lung Circ 22(11): 895-902.

5. Areskog M, Tibbling L, Wranne B (1980) Influence of coronary bypass surgery on oesophageal function and symptomatology. Acta Med Scand 207(5): 403-405.

6. Lenfant C (2010) Chest pain of cardiac and noncardiac origin. Metabolism 59 Suppl 1: S41-S46.

7. Kalisnik JM, Avbelj V, Trobec R, Gersak B (2007) Position-dependent changes in vagal modulation after coronary artery bypass grafting. Comput Biol Med 37(10): 1404-1408. 
8. Nalladaru Z, Wessels A, DuPreez L (2012) Tapia's syndrome--a rare complication following cardiac surgery. Interact Cardiovasc Thorac Surg 14(1): 131-132.

9. Seal D, Balaton J, Coupland SG, Eagle CJ, MacAdams C, et al. (1997) Somatosensory evoked potential monitoring during cardiac surgery: an examination of brachial plexus dysfunction. J Cardiothorac Vasc Anesth 11(2): 187-191.

10. Canbaz S, Turgut N, Halici U, Sunar H, Balci K, et al. (2005) Brachial plexus injury during open heart surgery--controlled prospective study. Thorac Cardiovasc Surg 53(5): 295-299.

11. Bordoni B, Marelli F (2015) The fascial system and exercise intolerance in patients with chronic heart failure: hypothesis of osteopathic treatment. J Multidiscip Healthc 8: 489-494

12. Bordoni B, Marelli F, Bordoni G (2016) A review of analgesic and emotive breathing: a multidisciplinary approach. J Multidiscip Healthc 9: 97-102.

13. Bordoni B, Marelli F (2016) Failed back surgery syndrome: review and new hypotheses. J Pain Res 9: 17-22.

14. Bordoni B, Zanier E (2015) The continuity of the body: hypothesis of treatment of the five diaphragms. J Altern Complement Med 21(4): 237-242.

15. Bordoni B, Zanier E (2013) Anatomic connections of the diaphragm: influence of respiration on the body system. J Multidiscip Healthc 6: 281-291.

16. Sanders RJ, Annest SJ (2014) Thoracic outlet and pectoralis minor syndromes. Semin Vasc Surg 27(2): 86-117.

17. Bordoni B, Zanier E (2011) Clinical and symptomatological reflections: the fascial system. J Multidiscip Healthc 7: 401-411.

18. Bordoni B, Zanier E (2015) Understanding Fibroblasts in Order to Comprehend the Osteopathic Treatment of the Fascia. Evid Based Complement Alternat Med 2015: 860934.

19. Chila AG (2010) Foundations for Osteopathic Medicine. ( $3^{\text {rd }}$ edn), Editor Lippincott Williams, USA.

20. Schleip R, Findley TW, Chaitow L, Huijing PA (2012) Fascia: The tensional network of the human body. Churchill Livingstone Elsevier, UK.

21. Goldstein FJ, Jeck S, Nicholas AS, Berman MJ, Lerario M (2005) Preoperative intravenous morphine sulfate with postoperative osteopathic manipulative treatment reduces patient analgesic use after total abdominal hysterectomy. J Am Osteopath Assoc 105(6) 273-279.

22. Wieting JM, Beal C, Roth GL, Gorbis S, Dillard L, et al. (2005) The effect of osteopathic manipulative treatment on postoperative medical and functional recovery of coronary artery bypass graft patients. J Am Osteopath Assoc 113(5): 384-393.

23. O-Yurvati AH, Carnes MS, Clearfield MB, Stoll ST, McConathy W] (2005) Hemodynamic effects of osteopathic manipulative treatment immediately after coronary artery bypass graft surgery. J Am Osteopath Assoc 105(10): 475-481.

24. Jarski RW, Loniewski EG, Williams J, Bahu A, Shafinia S, et al (2000) The effectiveness of osteopathic manipulative treatment as complementary therapy following surgery: a prospective, matchcontrolled outcome study. Altern Ther Health Med 6(5): 77-81.
25. Ruffini N, D’Alessandro G, Mariani N, Pollastrelli A, Cardinali L, et al. (2015) Variations of high frequency parameter of heart rate variability following osteopathic manipulative treatment in healthy subjects compared to control group and sham therapy: randomized controlled trial. Front Neurosci 9: 272.

26. Henley CE, Ivins D, Mills M, Wen FK, Benjamin BA (2008) Osteopathic manipulative treatment and its relationship to autonomic nervous system activity as demonstrated by heart rate variability: a repeated measures study. Osteopath Med Prim Care 2: 7.

27. Giles PD, Hensel KL, Pacchia CF, Smith ML. (2013) Suboccipital decompression enhances heart rate variability indices of cardiac control in healthy subjects. J Altern Complement Med 19(2): 92-96.

28. Cerritelli F, Carinci F, Pizzolorusso G, Turi P, Renzetti C, et al. (2011) Osteopathic manipulation as a complementary treatment for the prevention of cardiac complications: 12-Months follow-up of intima media and blood pressure on a cohort affected by hypertension. J Bodyw Mov Ther 15(1): 68-74.

29. Saggio G, Docimo S, Pilc J, Norton J, Gilliar W (2011) Impact of osteopathic manipulative treatment on secretory immunoglobulin a levels in a stressed population. J Am Osteopath Assoc 111(3): 143147

30. Uva B, Aliverti A, Bovio D, Kayser B (2016) The "Abdominal Circulatory Pump": An Auxiliary Heart during Exercise? Front Physiol 6: 411.

31. Rocha T, Souza H, Brandão DC, Rattes C, Ribeiro L, et al. (2015) The Manual Diaphragm Release Technique improves diaphragmatic mobility, inspiratory capacity and exercise capacity in people with chronic obstructive pulmonary disease: a randomised trial. J Physiother 61(4): 182-189.

32. Franke H, Hoesele K (2013) Osteopathic manipulative treatment (OMT) for lower urinary tract symptoms (LUTS) in women. J Bodyw Mov Ther 17(1): 11-18.

33. Mason DC, Ciervo CA (2009) Brachial plexus injuries in neonates: an osteopathic approach. J Am Osteopath Assoc 109(2): 87-91.

34. Sucher BM (1995) Placatory diagnosis and manipulative management of carpal tunnel syndrome: Part 2. 'Double crush' and thoracic outlet syndrome. J Am Osteopath Assoc 95(8): 471-479.

35. Bordoni B, Zanier E (2015) Sutherland's legacy in the new millennium: the osteopathic cranial model and modern osteopathy Adv Mind Body Med. Spring 29(2): 15-21.

36. Kasparian H, Signoret G, Kasparian J (2015) Quantification of Motion Palpation. J Am Osteopath Assoc 115(10): 604-610.

37. Martins WR, Diniz LR, Blasczyk JC, Lagoa KF, Thomaz S, et al. (2015) Immediate changes in electroencephalography activity in individuals with nonspecific chronic low back pain after cranial osteopathic manipulative treatment: study protocol of a randomized, controlled crossover trial. BMC Complement Altern Med 15: 223.

38. Masic I, Miokovic M, Muhamedagic B (2008) Evidence based medicine - new approaches and challenges. Acta Inform Med 16(4): 219-225.

39. Martingano D, Gurm H, Oliff A, Martingano FX, Aglialoro G (2012) Osteopathic Approach to the Diagnosis of Appendiceal Mucinous Cystadenocarcinoma Mimicking Primary Ovarian Malignant Neoplasm. J Am Osteopath Assoc 116(7): 480-484.

40. Chaitow L (2012) The ARTT of palpation? J Bodyw Mov Ther 16(2): 129-131. 
41. Shaw KA, Dougherty JJ, Treffer KD, Glaros AG (2012) Establishing the content validity of palpatory examination for the assessment of the lumbar spine using ultrasonography: a pilot study. J Am Osteopath Assoc 112(12): 775-782.
42. Elkiss ML, Jerome JA (2012) Touch--more than a basic science. J Am Osteopath Assoc 112(8): 514-517. 